\title{
Multicenter Study on the Diagnostic of Imaging Tests and EUS-guided fine needle aspiration of Solid Pseudopapillary Neoplasms of the Pancreas
}

\section{Vítor Doria Ricardo}

Irmandade da Santa Casa de Misericordia de Sao Paulo

Giulia Marchetti

Irmandade da Santa Casa de Misericordia de Sao Paulo

Arthur Ferraz de Almeida

Irmandade da Santa Casa de Misericordia de Sao Paulo

\section{César Vivian Lopes}

Santa Casa de Misericórdia de Porto Alegre: Santa Casa de Misericordia de Porto Alegre

Jerusa Santos dos Reis

Universidade Federal do Maranhão: Universidade Federal do Maranhao

\section{Eduardo Aimoré Bonin}

Universidade Federal do Paraná: Universidade Federal do Parana

Carlos Eduardo Gomes Callegari

Universidade Federal do Paraná: Universidade Federal do Parana

Wladimir Campos de Araújo

Hospital da Bahia

Marcel Autran Machado

Hospital 9 de Julho

Jose Celso ARDENGH ( $\nabla$ jcelso@uol.com.br)

Universidade de Sao Paulo Hospital das Clinicas da Faculdade de Medicina de Ribeirao Preto https://orcid.org/0000-0002-5932-2499

\section{Research Article}

Keywords: Endoscopic ultrasound, Endoscopic ultrasound-guided fine needle aspiration, Diagnosis, Pancreatic cancer, Pathology/Surgery, solid-pseudopapillary neoplasm

Posted Date: June 18th, 2021

DOI: https://doi.org/10.21203/rs.3.rs-614228/v1 
License: (c) (i) This work is licensed under a Creative Commons Attribution 4.0 International License. Read Full License 


\section{Abstract \\ Purpose}

Imaging diagnosis of SPN is difficult. Preoperative diagnosis by EUS-FNA) is possible, safe, and has been reported in the literature. However, its usefulness is still controversial. The aim of this study was to determine the accuracy of the EUS-FNA and imaging findings in CT and MRI/MRCP exams in the diagnosis of patients with SPN.

\section{Methods}

We retrospectively reviewed the medical records of patients undergoing EUS-FNA with suspected diagnosis of SPN on imaging studies in 5 high-volume hospitals. The final diagnosis was obtained after the histological examination of the surgical specimen. Demographic data, CT, MRI and EUS findings, anatomopathological specimen and $\mathrm{McH}$ results obtained by EUS were analyzed.

\section{Results}

Fifty-four patients were included, of which 49 (90.7\%) were women with an average age of 33.4 (range $11-78$ ) years. The most common symptom presented was abdominal pain, present in $35.2 \%$. SPN was detected incidentally in 32 (59\%). The mean size of the tumors was $3.8 \mathrm{~cm}$ (SD: 2.26$)$. The most common findings at EUS were a solid, solid/cystic, and cystic lesion in $52.9 \%, 41.1 \%$ and $7.8 \%$, respectively. The final diagnosis was SPN in 51 patients and NF-NET in 3. The correct diagnosis was made by CT, MRI, EUS and EUS-FNA in $21.9 \%, 28.9 \%, 64.7 \%$ and $88.2 \%$, respectively. EUS-FNA associated with CT and MRI increased diagnostic performance to $94.11 \%$ and $94.11 \%$, respectively.

\section{Conclusion}

Differential diagnosis between SPN and NF-NET with imaging tests can be difficult. EUS-FNA increases preoperative diagnosis in such cases and should be routinely used to rule out NF-NET.

\section{Introduction}

Pancreatic solid-pseudopapillary neoplasms (SPN) are rare and $90 \%$ occur in women in their 3rd and 4th decades of life [1, 2]. The symptoms are nonspecific or absent and such lesions are mostly incidentally diagnosed during imaging exams such as abdominal ultrasound (US), computerized tomography (CT) or magnetic resonance cholangiopancreatography (MRI/MRCP) [3]. SPN appears as a circumscribed, encapsulated, solid mass, but eventually with cystic areas [4]. Most patients have localized disease and up to $15 \%$ have metastasis or vascular invasion [5]. Conventional treatment is surgical resection, and 
unlike ductal pancreatic adenocarcinoma (PAC), the result is favorable with a five-year survival rate of $97 \%[1]$.

Accurate diagnosis of SPN is difficult based solely on imaging, especially in solid lesions, and can commonly mimic a non-functioning pancreatic neuroendocrine tumor (NF-NET), serous cystadenoma (SCA) and less commonly PAC [6]. Based on diagnostic imaging of a pancreatic nodule, SPN may be surgically removed without a preoperative diagnosis and previous anatomopathological confirmation. However, since such lesions are commonly found at the head of the pancreas, a duodenopancreatectomy (DP) procedure is associated to considerable morbidity and mortality rates.

Endosonography (EUS) is increasingly being used in the evaluation of pancreatic tumors. Recently, some studies evaluated the role in EUS-FNA in SPN [7-12], as well as comparing it with CT [13] and MRI for diagnosis, but its accuracy in SPN diagnosis remains unclear.

The aim of this study is to determine the diagnostic yield of imaging findings and EUS-FNA in the preoperative diagnosis of pancreatic solid cystic lesions.

\section{Methods}

This was a retrospective and multicentric case series, carried out in three reference University hospitals and two private tertiary referral hospitals in Brazil. The study was approved by the Research Ethics Committee of each institution and complied with the regulations of the Health Insurance Portability and Accountability Act (HI-PAA).

The study was approved by the Research Ethics Committee on 07/22/2019 under number: 3.518.999. A retrospective review was carried out at medical records of patients with suspected diagnosis of SPN at $\mathrm{CT}, \mathrm{MRI}$ and EUS or post-operative pathological evaluation. All selected patients signed an informed consent form before undergoing EUS-FNA. Age, sex, symptoms and CT, MRI and EUS-FNA findings were noted and the final diagnosis was obtained after the histological examination of the surgical specimen.

EUS was performed with conscious sedation or anesthesia monitored by an anesthesiologist. All procedures were performed by experienced doctors, always using a sectoral echoendoscope. All fineneedle biopsies were made under the same procedure. Fine-needle gauge specifications (19G, 22G, $25 \mathrm{G}$ and ProCore 20G), number of needles passes and micro histology $(\mathrm{McH})$ results, in addition to the surgical pathology results were recorded. The occurrence of adverse events (AE) was also documented.

\section{Sample handling}

Samples obtained by FNA were placed in 10\% formalin (6 to 24 hours) and followed the routine of the pathological anatomy sector of each center participating in the study. The blocks and biopsies embedded in paraffin were subjected to semi-series histological sections of $3 \mu \mathrm{m}$ thick, at three different depth levels, stained by the Hematoxylin-Eosin method. CellSens Micro Imaging Software (Olympus America Inc, 
Center Valley, PA) was used to dimension samples in millimeters. The diagnosis of SPN was confirmed through $\mathrm{McH}$ with hematoxylin-eosin and, when possible, immunohistochemistry (IHC). The sections stained with hematoxylin-Eosin (H / E) from both FNA and surgical samples were reviewed by specialized pathologists to confirm the diagnosis. The IHC markers (primary antibodies) used in the analysis included $\beta$-catenin, CD10, progesterone receptor (PR), CD99 and chromogranin A [14].

\section{Statistical analysis}

The collected and recorded data were stored in a database. The statistical analysis of continuous variables was described as mean and standard deviation and dichotomous variables expressed as simple proportions. The definitive diagnosis of SPN was based on histological analysis, and the patients who did not have it were excluded from the study. True positive cases were the ones that after undergoing CT, MRI, EUS and EUS-FNA, had the SPN diagnosis histologically confirmed. To assess the diagnostic gain acquired with the combination of imaging tests, the numbers of truly positive cases from each method were added. The comparison between the results obtained in the imaging exams was performed using the Mc Nemmar test for each pair of groups (CT + EUS; CT + EUS / FNA; MRI + EUS; MRI + EUS-FNA). The association between the size of the needle used and the diagnosis obtained in the $\mathrm{McH}$ was tested using Fisher's exact test. The level of statistical significance adopted was $95 \%$. The analyzes were performed using the STATA 14 software.

\section{Results}

All 54 patients had suspected SPN pancreatic tumors. Three patients had a non-functioning pancreatic neuroendocrine (NF-NET) tumor type G1 as a final diagnosis after surgical resection. The others $(n=51)$ had the diagnosis of SPN confirmed by $\mathrm{McH}$, anatomopathological report of the surgical specimen and IHC analysis. Of the 51 patients with confirmed SPN, $90.2 \%(n=46)$ were female and $9.8 \%(n=5)$ were male, and the mean age was 33.66 years (SD 14.46) (Table 1).

Twenty-one patients (41.2\%) were symptomatic, being the most common symptoms abdominal pain and weight loss associated with nausea or vomiting. Jaundice was present in $4(7.8 \%)$ patients. SPN was detected incidentally in 32 (59.3\%) of the patients. The average size of the cyst was $3.8 \mathrm{~cm}$ (SD 2.26) and the lesions were distributed between the body (20), head/uncinate (22), and tail (9) portions of the pancreas. One patient had two synchronous lesions identified on the body and tail of the pancreas. The solid, solid-cystic (microcystic) and cystic appearance was present in 27 (52.9\%), 21 (41.1\%) and 4 (7.8\%) cases, respectively (Figure 1). The lesion margin was well defined in $42(82.3 \%)$ of the cases. Calcification was present in $3(5.8 \%)$ patients. In $4(7.8 \%)$ patients there was vascular involvement detected by the EUS and confirmed by surgery, as well as dilation of the main pancreatic duct (MP) in $5(9.8 \%)$ patients (Table 1).

EUS-FNA was performed on all patients, without the pathologist presence of a rapid on site evaluation (ROSE) (Table 4). Conventional needles of 22G in 34 (66.66\%), 19G in 9 (17.64\%), Procore 20G in 5 $(9.8 \%)$ and $25 \mathrm{G}$ in $3(5.8 \%)$ were used. A median of 2.5 needle passes (range 1 to 5 ) was performed for 
each lesion. The diagnostic performance of the EUS-FNA was not affected by the size of the needle $(\mathrm{p}=$ 0.175). No adverse events were documented.

Surgical resection was performed in all patients who had no evidence of lymph nodes or distant metastases.

Pre-operative diagnostic yield of CT, MRI and EUS-FNA

CT was performed in 41 patients. Pancreatic cancer was suspected in 16 (39.02\%), SPN in 9 (21.95\%), pancreatic cystic lesion in $3(7.31 \%)$, nonfunctioning pancreatic neuroendocrine tumor (NF-NET) in 4 (9.75\%), chronic pancreatitis in $3(7.31 \%)$, serous cystadenoma (SCA) in $2(4.87 \%)$, hematoma after blunt trauma in $1(2.43 \%)$, common bile duct dilation (CBDD) in $1(2.43 \%)$ and pancreatic pseudocyst (PP) in 3 cases $(2.43 \%)$. The combination of CT + EUS and CT + EUS-FNA significantly increased $(p<0.005)$ the diagnosis compared to CT only, which went from $21.95 \%$ to $66.66 \%$ and $94.11 \%$, respectively (Table 2 ).

MRI / MRCP (Magnetic Resonance Cholangiopancreatography) was performed on 45 patients. Pancreatic cancer was suspected in 13 (28.88\%), SPN in 13 (28.88\%), pancreatic cystic lesion in 7 (15.55\%), nonfunctioning pancreatic neuroendocrine tumor (NF-NET) in $4(8.88 \%)$, serous cystadenoma in 2 (4.44\%), chronic pancreatitis in 1 (2.22\%), choledochal dilation in 1 (2.22\%), PP in $3(6.66 \%)$ and was normal in $1(2.22 \%)$ case. The combination of MRI / MRCP + EUS and MRI/MRCP + EUS-FNA significantly increased $(p<0.005)$ the diagnosis compared to MRI / MRCP only, which went from $28.88 \%$ to $66.66 \%$ and $94.11 \%$, respectively (Table 3 ).

EUS alone suspected the diagnosis of SPN in $33(64.71 \%)$. Two cases that were not included in this analysis because they received a final diagnosis of NF-NET, had a previous result of SPN through EUS. EUS suspected PAC in 9, NF-NET in 4 (7.8\%), chronic pancreatitis in 1, pancreatic cystic lesion in 1, serous cystadenoma in 2, and lymph node in 1 . The McH obtained by the EUS-FNA diagnosed SPN in 45 (88.24\%), NF-NET in 2 (3.92\%) and was negative in 4 cases (7.84\%), all confirmed as SPN after surgery.

\section{Discussion}

SPN are rare, with approximately 2744 reported cases [15]. US and CT are the most used imaging methods for diagnosis and represent approximately $80 \%$ of imaging studies for the diagnosis of SPN. In the past few decades, the use of MRI has increased substantially, as has EUS, but it still accounts for only $5 \%$ of the imaging methods used for the diagnosis of SPN. In addition, there are few studies that determine the additional benefit offered by the EUS-FNA to imaging tests such as CT and MRI [16]. The benefit in the use of EUS in patients with solid and cystic pancreatic tumors with a yield approaching $78.8 \%$ and $71.4 \%$, respectively, has been proven $[17,18]$. Therefore, the inclusion of EUS-FNA increases the diagnostic accuracy of this type of tumor.

The results of this study highlight the difficulty of CT, MRI and EUS in correctly classifying the SPN using only the image resources, with a diagnostic performance of $21.95 \%, 28.88 \%, 64.71 \%$ respectively. These 
results are similar to another recent study, which was $23.5 \%$ and $41.2 \%$ for CT and EUS, respectively [13]. When we include the results of EUS-FNA, there is an increase in the diagnostic performance of CT (72.16\%) and MRI (65.23\%), rising up to $94.11 \%$ in both methods.

Classically, SPN are described as circumscribed, hypoechoic, solid, heterogeneous masses and eventually with a cystic component. However, this appearance presents in just $60 \%$ of patients, with a predominantly solid lesion found in $32 \%$ of cases, suggesting a more classic appearance associated with a neuroendocrine tumor [19]. In our series, the solid, solid/cystic, and cystic morphology was $52.9 \%, 41.1 \%$ and $7.8 \%$ respectively. SPN can mimic other pancreatic tumors, which can lead to diagnostic challenges [20].

This fact can be observed in our study, as CT and EUS suspected the presence of NF-NET in (9.75\%) and (7.8\%), respectively, since the injuries identified by these exams had a predominantly solid component. When microcystic, SPN are similar to serous cystadenoma and can cause diagnostic confusion, which occurred in $4 \%$ of the cases examined by CT, MRI and EUS. The microcystic pattern must be recognized as part of the morphological spectrum of the SPN, which can lead to confusion regarding the presence of a serous cystadenoma [6]. Previous CT and EUS studies, in which SPN was identified as other cystic lesions in the pancreas in up to $50 \%$ of cases, including benign lesions such as serous cystadenoma, confirm the findings of our study $[13,21,22]$.

Some cases were interpreted as pseudocysts, highlighting the difficulty in determining the etiology of a cystic lesion based only on morphological characteristics of the image. This occurred in $7.31 \%$ and $15.55 \%$ of CTs and MRIs, respectively. Therefore, the importance of correctly classifying SPN is emphasized in this study, where $8(33.3 \%)$ cysts were erroneously classified by CT, as pseudocysts (2), unspecific pancreatic cystic lesion (2), unspecific pancreatic cystic lesion with calcification (2), hematoma (1) and serous cystadenoma (1), all which have prognosis and management completely different from those adopted in SPN. The same happened with MRI / MRCP which was normal in 3 patients and identified unspecific pancreatic cystic lesion in 4 and pseudocyst in 3 patients.

In the literature, rupture of the SPN is associated with abdominal trauma or may be spontaneous, which is rare. To date, there have been only 3 cases of SPN with spontaneous rupture reported worldwide [23]. In our series, we had two young patients with blunt abdominal trauma who underwent imaging exams that suspected hematoma (1) and pseudocyst (1). In these two cases, the EUS-FNA was crucial for the diagnosis of SPN, changing the management of these patients.

The aim of this study was to confirm the benefit of performing the EUS-FNA to increase the diagnostic performance of other conventional imaging tests such as CT and MRI. The EUS-FNA association increased diagnostic yield by almost $72.16 \%$ for CT and $65.23 \%$ for MRI. The results are similar to previous studies, which report a low sensitivity rate and absence of adverse events as in the current series [13]. Regarding the adverse events resulting from the EUS-FNA, the first case of neoplastic cell implantation in the stomach was recently described [24]. In our series, even after prolonged follow-up and interviews with patients and attending physicians, we did not observe this type of complication. 
In the present series we found 2 cases of interest, as they had a strong suspicion of SPN on imaging exams. In the first, CT and MRI confirmed the diagnosis of pseudocyst, the EUS suspected SPN, and the EUS-FNA revealed NF-NET, which was confirmed by uncinate process resection. The second had CT, MRI and EUS image exams with SPN morphology (solid-cystic), the McH obtained by EUS-FNA confirmed the presence of SPN, but the operative piece confirmed NF-NET. In this case, the diagnosis by McH confirming SPN was performed only by HE, as there was not enough material to perform the IHC. These findings corroborate those found in the literature where the EUS-FNA performed on suspected SPN has excellent positive and negative predictive value, however the most common classification errors were with NF-NET which presents no clinical impact, just as it was observed in our series [8]. In addition, in 177 patients studied by the EUS-FNA with suspected NF-NET, discrepancies were reported in 14 patients. In 4 of them the erroneous diagnosis was SPN. Accordingly, it is concluded that when an adequate sample is obtained, the most significant error is the incorrect classification, which is more often associated with SPN, but the damage associated with this diagnostic error is minimal [25].

The authors conclude that the isolated CT, MRI and EUS have low rates of correct diagnosis, but the association with EUS-FNA increases preoperative diagnosis and should be considered to rule out other pancreatic diseases such as NF-NET and SCA. These results suggest that patients who do not have a clear diagnosis of SPN, as is usually the case, should undergo EUS-FNA.

\section{Declarations}

Funding: This study did not obtain any funding.

Conflicts of interest / competing interests: All authors declare that there is no conflict of interest.

Availability of data and materials: Data from each of the patients included in this study are and will be available at any time for further investigation.

\section{Code availability: not applicable}

Author's contributions: Author Contributions:

\section{Category 1}

Conception and design of study: M. A. Machado, J. C. Ardengh.

Acquisition of data: V.D. Ricardo, G.Marchetti, A. F. de Almeida.

Analysis and/or interpretation of data: C. V. Lopes, J. dos Santos-Reis, E. A. Bonin, W. C. de Araújo.

\section{Category 2}

Drafting the manuscript: A. F. de Almeida, and J. C. Ardengh. 
Revising the manuscript critically for important intellectual content: V.D. Ricardo, G. Marchetti, A. F. de Almeida, C. V. Lopes, J. dos Santos-Reis, C. E. G. Callegari, E. A. Bonin, W. C. de Araújo, M. A. Machado, J. C. Ardengh.

\section{Category 3}

Approval of the version of the manuscript to be published (the names of all authors must be listed):

V.D. Ricardo, G. Marchetti, A. F. de Almeida, C. V. Lopes, J. dos Santos-Reis, C. E. G. Callegari, E. A. Bonin, W. C. de Araújo, M. A. Machado, J. C. Ardengh.All the authors mentioned above contributed equally to the preparation of this study. However, the methodological design was conceived by the last author.

Ethics approval: The study was approved by the Research Ethics Committee of each institution and complied with the regulations of the Health Insurance Portability and Accountability Act (HI-PAA). The study was approved by the Research Ethics Committee of the proposing institution on 07/22/2019 under number: $3,518,999$, followed by the evaluation of co-participating centers.

Acknowledgement: None to declare.

Consent to Publication: All authors included in this study agree to publish this article in the International Journal of Gastrointestinal Cancer if accepted for publication.

\section{References}

1. Klimstra DS, Wenig BM, Heffess CS. Solid-pseudopapillary tumor of the pancreas: a typically cystic carcinoma of low malignant potential. Semin Diagn Pathol. 2000;17(1):66-80. https://www.ncbi.nlm.nih.gov/pubmed/10721808.

2. Machado MC, Machado MA, Bacchella T, et al. Solid pseudopapillary neoplasm of the pancreas: distinct patterns of onset, diagnosis, and prognosis for male versus female patients. Surgery. 2008;143(1):29-34. https://doi.org/10.1016/j.surg.2007.07.030.

3. Palmucci S, Uccello A, Leone G, Failla G, Ettorre GC. Rare pancreatic neoplasm: MDCT and MRI features of a typical solid pseudopapillary tumor. J Radiol Case Rep. 2012;6(1):17-24. https://doi.org/10.3941/jrcr.v6i1.823.

4. McCluney S, Wijesuriya N, Sheshappanavar V, Chin-Aleong J, Feakins R, Hutchins R, et al. Solid pseudopapillary tumour of the pancreas: clinicopathological analysis. ANZ J Surg. 2018;88(9):8915. https://doi.org/10.1111/ans.14362.

5. Vollmer CM Jr, Dixon E, Grant DR. (2003) Management of a solid pseudopapillary tumor of the pancreas with liver metastases. HPB (Oxford) 2003;5(4):264-7. https://doi.org/10.1080/13651820310001397.

6. Abe A, Ohishi Y, Miyazaki T, Ozono K, Mochidome N, Saeki K, et al. 'Microcystic pattern' should be recognised as part of the morphological spectrum of solid-pseudopapillary neoplasm of the 
pancreas. Histopathology. 2018;72(2):216-26. https://doi.org/10.1111/his.13376.

7. Karsenti D, Caillol F, Chaput U, Perrot B, Koch S, Vuitton L, et al. Safety of Endoscopic UltrasoundGuided Fine-Needle Aspiration for Pancreatic Solid Pseudopapillary Neoplasm Before Surgical Resection: A European Multicenter Registry-Based Study on 149 Patients. Pancreas. 2020;49(1):348. https://doi.org/10.1097/MPA.0000000000001460.

8. Hooper K, Tracht JM, Eldin-Eltoum IA. Cytologic criteria to reduce error in EUS-FNA of solid pseudopapillary neoplasms of the pancreas. J Am Soc Cytopathol. 2017;6(6):228-35. https://doi.org/10.1016/j.jasc.2017.06.002.

9. Mirminachi B, Farrokhzad S, Sharifi AH, Nikfam S, Nikmanesh A, Malekzadeh R, et al. Solid Pseudopapillary Neoplasm of Pancreas; A Case Series and Review Literature. Middle East J Dig Dis. 2016;8(2):102-8. https://doi.org/10.1016/j.jasc.2017.06.002.

10. Yamaguchi M, Fukuda T, Nakahara M, Amano M, Takei D, Kawashima M, et al. Multicentric solid pseudopapillary neoplasms of the pancreas diagnosed by endoscopic ultrasound-guided fine needle aspiration: a case report. Surg Case Rep. 2015;1(1):110. https://doi.org/10.1186/s40792-015-0111-8.

11. Jahangir S, Loya A, Siddiqui MT, Akhter N, Yusuf MA. Accuracy of diagnosis of solid pseudopapillary tumor of the pancreas on fine needle aspiration: A multi-institution experience of ten cases. Cytojournal. 2015;12:29. https://doi.org/10.4103/1742-6413.171140.

12. Hosokawa I, Shimizu H, Ohtsuka M, Kato A, Yoshitomi H, Furukawa K, et al. Preoperative diagnosis and surgical management for solid pseudopapillary neoplasm of the pancreas. J Hepatobiliary Pancreat Sci. 2014;21(8):573-8. https://doi.org/10.1002/jhbp.96.

13. Law JK, Stoita A, Wever W, Gleeson FC, Dries AM, Blackford A, et al. Endoscopic ultrasound-guided fine needle aspiration improves the pre-operative diagnostic yield of solid-pseudopapillary neoplasm of the pancreas: an international multicenter case series (with video). Surg Endosc. 2014;28(9):2592-8. https://doi.org/10.1007/s00464-014-3508-8.

14. Ardengh JC, Lopes CV, de Lima LF, Venco F, Santo GC, Begnami MD, et al. Cell block technique and cytological smears for the differential diagnosis of pancreatic neoplasms after endosonographyguided fine-needle aspiration. Acta Gastroenterol Latinoam. 2008;38(4):246-51. https://www.ncbi.nlm.nih.gov/pubmed/19157379.

15. Law JK, Ahmed A, Singh VK, Akshintala VS, Olson MT, Raman SP, et al (2014) A systematic review of solid-pseudopapillary neoplasms: are these rare lesions? Pancreas 43(3):331-7. https://doi.org/10.1097/MPA.0000000000000061.

16. Khashab MA, Kim K, Lennon AM, Shin EJ, Tignor AS, Amateau SK, et al. Should we do EUS/FNA on patients with pancreatic cysts? The incremental diagnostic yield of EUS over CT/MRI for prediction of cystic neoplasms. Pancreas. 2013;42(4):717-21. https://doi.org/10.1097/MPA.0b013e3182883a91.

17. Ardengh JC, Lopes CV, de Lima LF, de Oliveira JR, Venco F, Santo GC, et al. Diagnosis of pancreatic tumors by endoscopic ultrasound-guided fine-needle aspiration. World J Gastroenterol. 2007;13(22):3112-6. https://doi.org/10.1590/s0004-28032013000100003. 
18. Vaiciunas S, Taglieri E, Micelli-Neto O, Brunaldi MO, Venco F, Goldman SM, et al. Endoscopic Ultrasound-Guided Fine-Needle Aspiration Microhistology in Asymptomatic and Symptomatic Pancreatic Cystic Lesions. Pancreas. 2020;49(4):584-90. https://doi.org/10.1097/MPA.0000000000001525.

19. De Robertis R, Marchegiani G, Catania M, Ambrosetti MC, Capelli P, Salvia R, et al. Solid Pseudopapillary Neoplasms of the Pancreas: Clinicopathologic and Radiologic Features According to Size. AJR Am J Roentgenol. 2019;213(5):1073-80. https://doi.org/10.2214/AJR.18.20715.

20. Bhatnagar R, Olson MT, Fishman EK, Hruban RH, Lennon AM, Ali SZ. Solid-pseudopapillary neoplasm of the pancreas: cytomorphologic findings and literature review. Acta Cytol. 2014;58(4):347-55. https://doi.org/10.1159/000363546.

21. Kang CM, Kim KS, Choi JS, Kim H, Lee WJ, Kim BR. Solid pseudopapillary tumor of the pancreas suggesting malignant potential. Pancreas. 2006;32(3):276-80. https://doi.org/10.1097/01.mpa.0000202956.41106.8a.

22. Yu MH, Lee JY, Kim MA, Kim SH, Lee JM, Han JK, et al. MR imaging features of small solid pseudopapillary tumors: retrospective differentiation from other small solid pancreatic tumors. AJR Am J Roentgenol. 2010;195(6):1324-32. https://doi.org/10.2214/AJR.10.4452.

23. Xu X, Chen D, Cao L, Feng X, Tong R, Zheng S, et al. Spontaneous rupture of solid pseudopapillary tumor of pancreas: A case report and review of literature. Medicine. 2019;98(44):e17554. https://doi.org/10.1097/MD.0000000000017554.

24. Yamaguchi H, Morisaka H, Sano K, Nagata K, Ryozawa S, Okamoto K, et al. Seeding of a Tumor in the Gastric Wall after Endoscopic Ultrasound-guided Fine-needle Aspiration of Solid Pseudopapillary Neoplasm of the Pancreas. Intern Med. 2020;59(6):779-82.

https://doi.org/10.2169/internalmedicine.3244-19.

25. Hooper K, Mukhtar F, Li S, Eltoum IA. Diagnostic error assessment and associated harm of endoscopic ultrasound-guided fine-needle aspiration of neuroendocrine neoplasms of the pancreas. Cancer Cytopathol. 2013;121(11):653-60. https://doi.org/10.1002/cncy.21332.

\section{Tables}

Table 1. Characteristics of patients submitted to EUS-FNA with suspected diagnosis of SPN on imaging exams in 5 different endoscopy centers in Brazil between January 1997 and January 2020 


\begin{tabular}{|c|c|c|}
\hline Variables & $\mathbf{N}$ & $\%$ \\
\hline \multicolumn{3}{|l|}{ Sex } \\
\hline Female & 46 & 90.2 \\
\hline Male & 5 & 9.8 \\
\hline Age (average/SD) & 33.66 & 14.46 \\
\hline \multicolumn{3}{|l|}{ Symptoms } \\
\hline Asymptomatic & 30 & 58.8 \\
\hline Abdominal pain & 18 & 35.2 \\
\hline Weight loss & 6 & 11.7 \\
\hline Nausea/vomiting & 7 & 13.7 \\
\hline Jaundice & 4 & 7.8 \\
\hline Acute pancreatitis & 2 & 3.9 \\
\hline Blunt trauma & 2 & 3.9 \\
\hline Cyst size (mm) (average/SD) & 3.87 & 2.26 \\
\hline \multicolumn{3}{|l|}{ Cyst location } \\
\hline Head & 22 & 43.14 \\
\hline Body & 20 & 39.22 \\
\hline Tail & 9 & 17.65 \\
\hline \multicolumn{3}{|l|}{ Echogenicity } \\
\hline Solid & 27 & 52.9 \\
\hline Solid/cystic & 21 & 41.1 \\
\hline Cystic & 4 & 7.8 \\
\hline \multicolumn{3}{|l|}{ Margin } \\
\hline Well defined & 42 & 82.3 \\
\hline Infiltrative & 9 & 17.7 \\
\hline Calcification & 3 & 5.8 \\
\hline \multicolumn{3}{|l|}{ Extra pancreatic spread } \\
\hline Vascular involvement & 4 & 7.8 \\
\hline Lymph node involvement & 0 & 0 \\
\hline
\end{tabular}




\begin{tabular}{|lll|}
\hline Liver metastases & 0 & 0 \\
\hline Dilated main pancreatic duct, $\mathbf{n}(\%)$ & 5 & 9.8 \\
\hline
\end{tabular}

Table 2. Performance of imaging exams, including $\mathrm{CT}$, in relation to the total number of cases that were submitted to each group of imaging exams and that later proved to be truly positive through post-surgical histological diagnosis.

\begin{tabular}{lllll} 
& CT only & CT + EUS & CT + EUS/FNA & p-value \\
\hline Truly positive test & 9 & 34 & 48 & $<0.005$ \\
\hline Truly positive cases & 41 & 51 & 51 & \\
\hline Total & $21.95 \%$ & $66.66 \%$ & $94.11 \%$ &
\end{tabular}

Table 3. Performance of imaging tests, including magnetic resonance imaging, in relation to the total number of cases that were submitted to each group of imaging exams and that later proved to be truly positive through post-surgical histological diagnosis.

\begin{tabular}{lllll}
\hline & MRI only & MRI + EUS & MRI + EUS/FNA & p-value \\
\hline Truly positive test & 13 & 34 & 48 & $<0.005$ \\
\hline Truly positive cases & 45 & 51 & 51 & \\
\hline Total & $28.89 \%$ & $66.66 \%$ & $94.11 \%$ & \\
\hline
\end{tabular}


Table 4. Relationship between the gauge used to perform fine needle aspiration and the final histopathological result in patients with suspected SPN from 5 different endoscopy centers in the city of São Paulo between January 1997 and January 2020

\begin{tabular}{rlll}
\hline & EUS-FNA MCH + & EUS-FNA MCH - & p-value \\
\hline Needle (G) & & & 0.175 \\
\hline 19 & $6(13.33 \%)$ & $3(50 \%)$ & \\
\hline 20 & $5(11.11 \%)$ & $0(0)$ & \\
\hline 22 & $31(68.89 \%)$ & $3(50 \%)$ & \\
\hline 25 & $3(6.67 \%)$ & $0(0)$ &
\end{tabular}

$\mathrm{MCH}$, microhistology; +, positive; -, negative; G, Gauge;

\section{Figures}

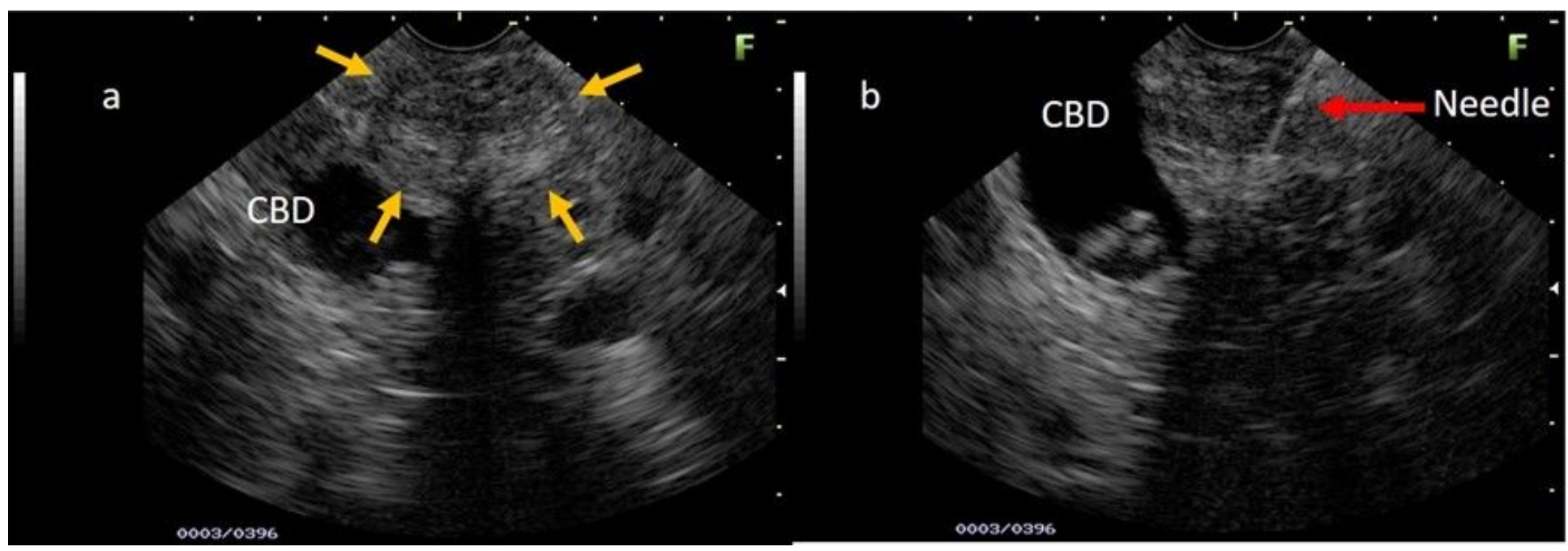

Figure 1

(a) Endosonography image of a young patient with a hypoechoic area (yellow arrows), also heterogeneous with imprecise limits and a microcystic component, right next to the common bile duct (CBD) with stones inside ( $a$ and $b$ ). The endosonography images suspected SPN. (b) Moment of the EUSFNA (red arrow), with the needle (22G) positioned in the center of the lesion. The biopsy revealed chronic inflammatory process without evidence of SPN. 


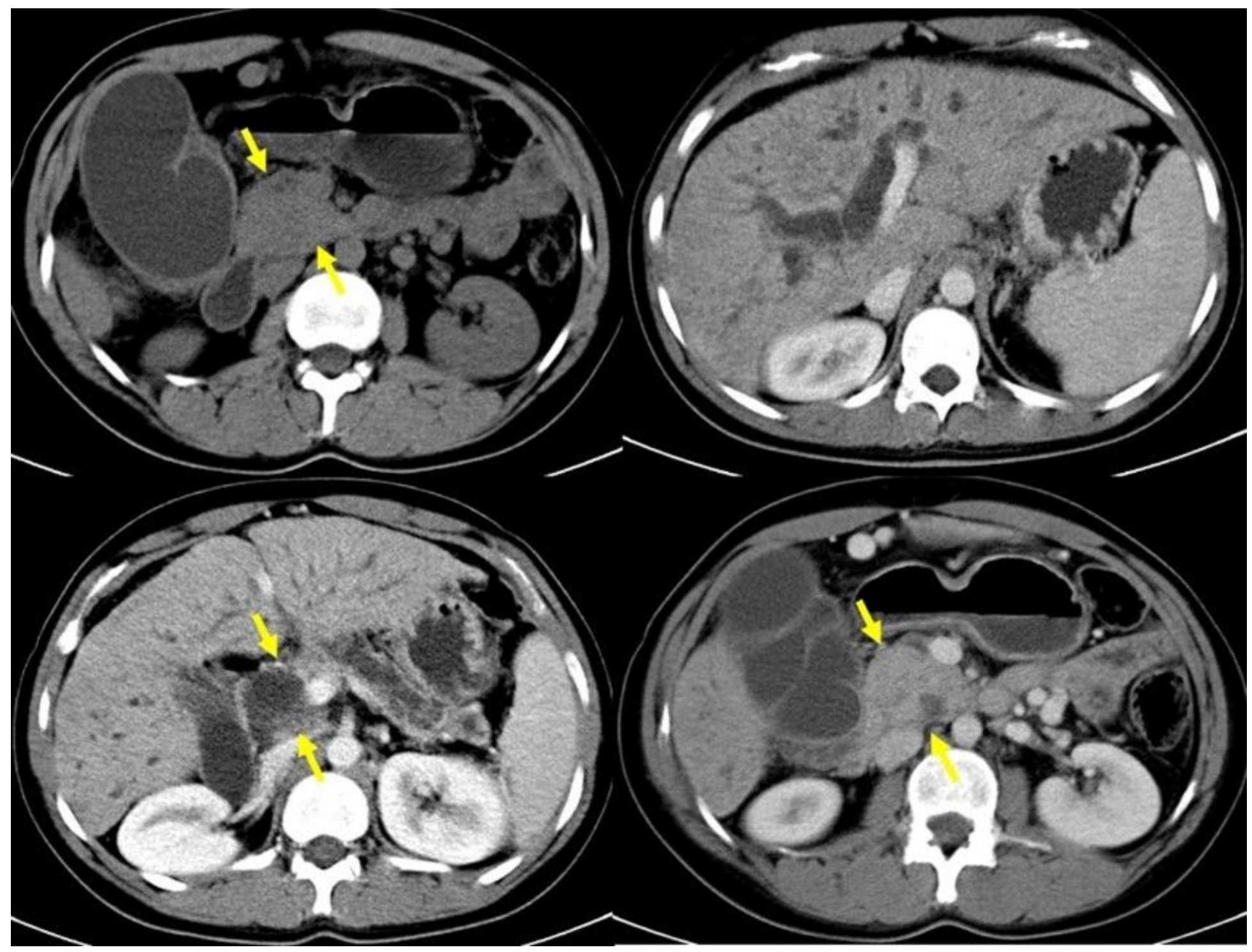

Figure 2

CT images with enlarged pancreatic head, dilation of the main pancreatic and choledochal duct, in addition to atrophy of the pancreatic gland in a 23-year-old woman with a history of chronic alcoholism (same case in figure 3). 


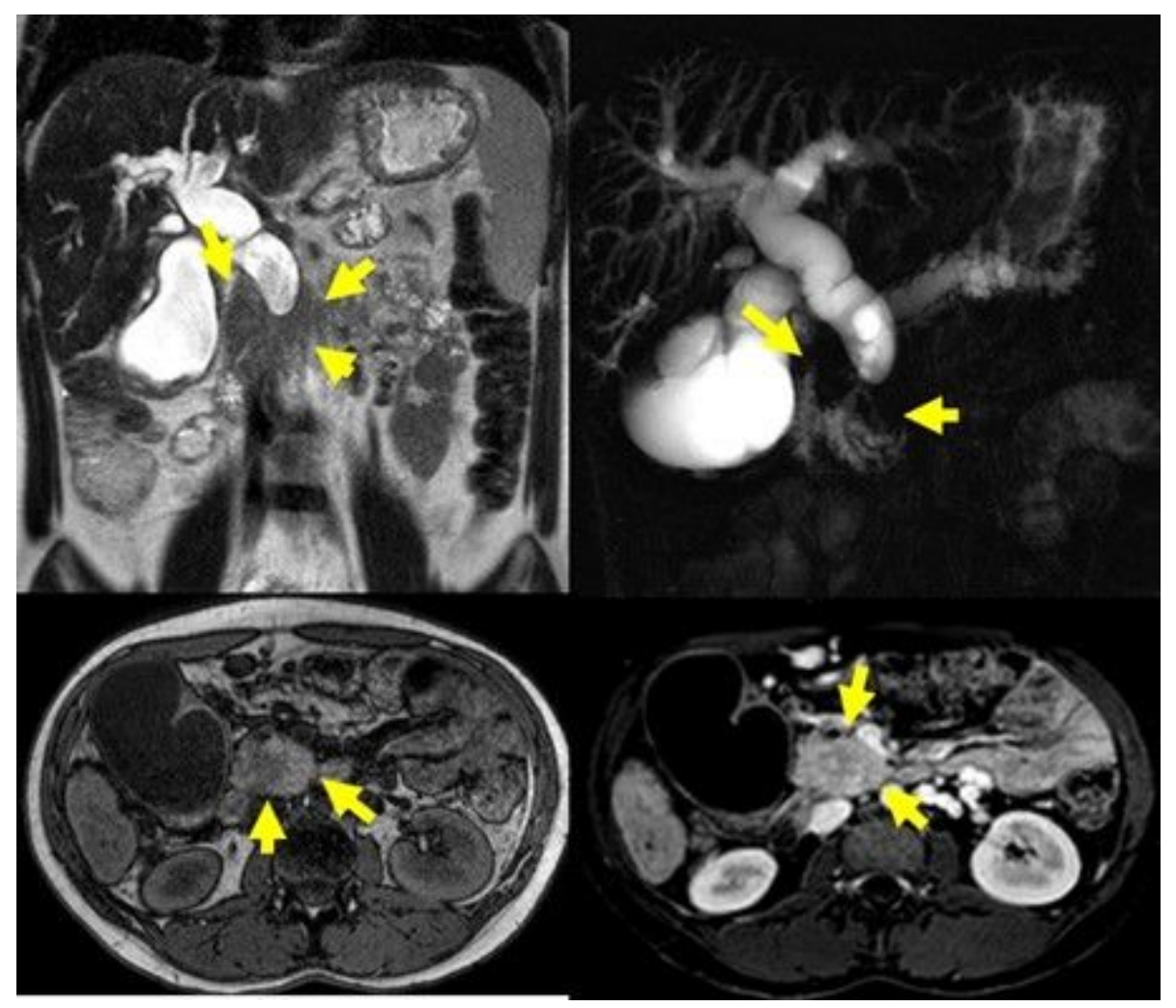

Figure 3

23-year-old woman with signs of chronic pancreatitis on MRI / MRCP and enlarged pancreatic head. 